\title{
Prevalência de diabetes mellitus tipo 2 e outros fatores de risco associados em pacientes com glaucoma
}

\author{
Prevalence of type 2 diabetes mellitus and other \\ associated risk factors in glaucoma patients
}

Jacqueline Coblentz ${ }^{1}$, Maria Lucia Elias Pires², Diogo Oliveira ${ }^{3}$, Karina Lebeis ${ }^{4}$, Larissa Terrezo ${ }^{5}$

\section{Resumo}

Objetivo: Avaliar a prevalência de diabéticos em uma amostra de pacientes com glaucoma; verificar se existe associação entre diabetes mellitus e glaucoma na amostra estudada; verificar outros fatores de risco associados. Métodos: Foram analisados de forma retrospectiva os prontuários de 50 pacientes com diagnóstico de glaucoma. Os dados registrados foram sexo, idade, raça, história familiar de glaucoma e história pessoal de diabetes mellitus tipo 2. Resultados: Do total de pacientes avaliados, 5 (10\%) apresentavam diabetes mellitus tipo 2. Destes, 3 eram mulheres e 2 eram homens, com mediana de idade de 81 anos (71-88). A prevalência de diabetes nos pacientes com glaucoma não mostrou diferenças significativas (OR: 1,476; Intervalo de Confiança $95 \%: 0,4438 \mathrm{a} 4,910 ; \mathrm{p}=0,5352)$ quando comparada à prevalência de diabetes mellitus tipo 2 na população geral brasileira $(7,6 \%)$. Conclusão: Nesta amostra de pacientes com glaucoma, a prevalência de diabetes mellitus tipo 2 foi pouco mais elevada que a da população. Entretanto, nenhuma associação foi encontrada entre diabetes mellitus e glaucoma.

Descritores: Glaucoma; Fatores de risco; Glaucoma/epidemiologia; Prevalência; Diabetes mellitus

\footnotetext{
${ }^{1}$ Médica oftalmologista da Universidade Federal do Estado do Rio de Janeiro - UNIRIO - Rio de Janeiro (RJ), Brasil;

${ }^{2}$ Professora associada da Disciplina de Clínica Médica III da Universidade Federal do Estado do Rio de Janeiro - UNIRIO - Rio de Janeiro (RJ), Brasil;

${ }^{3}$ Acadêmico do $3^{\circ}$ Ano da Faculdade de Medicina da Universidade Federal do Estado do Rio de Janeiro - UNIRIO - Rio de Janeiro (RJ), Brasil;

${ }^{4}$ Acadêmica do $3^{\circ}$ Ano da Faculdade de Medicina da Universidade Federal do Estado do Rio de Janeiro - UNIRIO - Rio de Janeiro (RJ), Brasil;

${ }^{5}$ Acadêmica do $3^{\circ}$ Ano da Faculdade de Medicina da Universidade Federal do Estado do Rio de Janeiro - UNIRIO - Rio de Janeiro (RJ), Brasil.
}

Trabalho realizado no Hospital Universitário Gaffrée e Guinle da Universidade Federal do Estado do Rio de Janeiro - UNIRIO - Rio de Janeiro (RJ), Brasil. 


\section{INTRODUÇÃO}

$\mathbf{O}$ glaucoma primário de ângulo aberto (GPAA) é a principal causa de cegueira ${ }^{(1)}$. Os fatores de risco comumente aceitos para GPAA incluem idade avançada, pressão intraocular elevada, história familiar positiva para glaucoma e raça ${ }^{(2)}$.

A relação entre diabetes mellitus (DM) e glaucoma não é bem estabelecida ${ }^{(2)}$. Entretanto, diversos estudos encontraram associação positiva entre diabetes e glaucoma, enquanto outros falharam ao tentar confirmar esta associação $\mathrm{O}^{(3-8)}$.

Embora a relação entre DM e GPAA seja controversa, uma metanálise de estudos seccionais sugeriu que o DM está associado a risco 1,5 vezes maior de $\mathrm{GPAA}^{(9)}$.

Os objetivos deste estudo foram: avaliar a prevalência de diabéticos em uma amostra de pacientes com glaucoma; verificar se existe associação entre diabetes mellitus e glaucoma na amostra estudada; verificar outros fatores de risco associados.

\section{Métodos}

Foram analisados de forma retrospectiva os prontuários de 50 pacientes com diagnóstico de glaucoma, acompanhados no ambulatório de oftalmologia do Hospital Universitário Gaffrée e Guinle da Universidade Federal do Estado do Rio de Janeiro (HUGG-UNIRIO), no período de julho de 2005 a julho de 2006.

Foram excluídos prontuários com informações incompletas. Os dados registrados foram sexo, idade, raça, história familiar de glaucoma e história pessoal de diabetes mellitus tipo 2 .

Para análise dos dados foi utilizado o programa GraphPad Instat ${ }^{\circledR}$, versão 3.1. Os resultados foram apresentados como média \pm desvio-padrão (variáveis com distribuição normal) e mediana e amplitude (dados sem distribuição normal).

A associação entre diabetes e glaucoma foi testada pelo teste Exato de Fisher e o grau de associação, pela medida de associação Odds Ratio e seu intervalo de confiança de $95 \%$.

Por não termos grupo controle, a comparação foi feita com os dados calculados em nosso meio, estabelecidos na literatura, para a frequência de diabetes mellitus tipo 2 na população geral.

\section{Resultados}

A amostra foi constituída de 20 (40\%) mulheres e 30 (60\%) homens, 18 (36\%) brancos e 32 (64\%) não- brancos (23 pardos e 9 negros).

A idade variou entre 30 e 88 anos, com média de $63,28 \pm 12,86$ anos.

Oito $(16 \%)$ pacientes tinham história familiar positiva para glaucoma.

Do total de pacientes avaliados, $5(10 \%)$ apresentavam diabetes mellitus tipo 2. Destes, 3 eram mulheres e 2 eram homens, com mediana de idade de 81 anos (71-88).

A prevalência de diabetes nos pacientes com glaucoma não mostrou diferenças significativas (OR: 1,476; Intervalo de Confiança 95\%: 0,4438 a 4,910; $p=$ $0,5352)$ quando comparada à prevalência de diabetes mellitus tipo 2 na população geral brasileira $(7,6 \%)$.

Os fatores de risco que predominaram foram raça (negros e pardos) e idade avançada.

\section{Discussão}

A prevalência de diabetes mellitus no grupo estudado foi de $10 \%$. Como não havia grupo controle, esse valor foi comparado a prevalência de DM na população geral. Não houve diferença significativa entre o grupo estudado e a população geral brasileira.

Chopra et al. (The Los Angeles Latino Eye Study) avaliaram 5.894 pacientes latinos acima de 40 anos, consecutivamente, para a presença de DM e/ou glaucoma. Destes, 1157 (19,6\%) tinham DM e 288 (4,9\%) tinham glaucoma. Os resultados mostraram que a prevalência de glaucoma foi $40 \%$ maior nos diabéticos, e que a presença de diabetes não mostrou associação com maior risco de glaucoma ${ }^{(2)}$.

Ainda não existe consenso na literatura no que se refere ao diabetes como fator de risco para desenvolvimento de glaucoma primário de ângulo aberto.

Nosso estudo não mostrou diferença significativa na prevalência de diabetes nos pacientes portadores de glaucoma, quando comparados à população geral. Entretanto, novos estudos são necessários para que se esclareça este fator de risco controverso até então.

\section{ConClusão}

Nesta amostra de pacientes com glaucoma, a prevalência de diabetes mellitus tipo 2 foi pouco mais elevada que a da população. Entretanto, nenhuma associação foi encontrada entre diabetes mellitus e glaucoma. São necessários outros estudos com amostras maiores para ser observada a possível associação entre glaucoma e diabetes, uma vez que não há consenso na literatura sobre este possível fator de risco. 


\begin{abstract}
Purpose: To evaluate prevalence of diabetes mellitus in a group of patients with glaucoma; to verify if there is association between diabetes mellitus and glaucoma; to verify other associated risk factors. Methods: Fifty (50) glaucoma patients had their medical records analyzed in a retrospective way. Registered data included sex, age, ethnic group, family history of glaucoma and personal history of type 2 diabetes mellitus. Results: Five (10\%) of all evaluated patients had type 2 diabetes mellitus. 3 of them were female and 2 were male, median age of 81 years old (71-88). Prevalence of diabetes in glaucoma patients did not show significant differences. (OR:1.476;95\% Confidence Interval:0.4438 to 4.910; $p=0.5352)$ when compared to the prevalence of type 2 diabetes mellitus in general brazilian population (7.6\%). Conclusion: In this group of patients with glaucoma, prevalence of type 2 diabetes mellitus was slightly higher than in general population. However, no association was found between diabetes mellitus and glaucoma.

Keywords: Glaucoma; Risk factors;Glaucomal epidemiology; Prevalence; Diabetes mellitus
\end{abstract}

\title{
RefERÊNCIAS
}

1. Leske MC. The epidemiology of open-angle glaucoma: a review. Am J Epidemiol. 1983;118(2):166-91.

2. Chopra V, Varma R, Francis BA, Wu J, Torres M, Azen SP; Los Angeles Latino Eye Study Group. Type 2 diabetes mellitus and the risk of open-angle glaucoma the Los Angeles Latino Eye Study. Ophthalmology. 2008;115(2):227-32.e1.

3. Quigley HA, West SK, Rodriguez J, Munoz B, Klein R, Snyder $\mathrm{R}$. The prevalence of glaucoma in a population-based study of Hispanic subjects: Proyecto VER. Arch Ophthalmol. 2001;119(12):1819-26.

4. Tielsch JM, Katz J, Quigley HA, Javitt JC, Sommer A. Diabetes, intraocular pressure, and primary open-angle glaucoma in the Baltimore Eye Survey. Ophthamology. 1995;102(1):48-53.

5. Wormald RP, Basauri E, Wright LA, Evans JR. The African Caribbean Eye Survey: risk factors for glaucoma in a sample of African Caribbean people living in London. Eye. 1994;8(Pt 3):315-20.

6. Kaimbo DK, Buntinx F, Missotten L. Risk factors for openangle glaucoma: a case-control study. J Clin Epidemiol. 2001;54(2):166-71.

7. Charliat G, Jolly D, Blanchard F. Genetic risk factor in primary open-angle glaucoma: a case-control study. Ophthalmic Epidemiol. 1994;1(3):131-8.

8. Wilson MR, Hertzmark E, Walker AM, Childs-Shaw K, Epstein DL. A case-control study of risk factors in open-angle glaucoma. Arch Ophthalmol. 1987;105(8):1066-71.

9. Bonovas S, Peponis V, Filioussi K. Diabetes mellitus as a risk factor for primary open-angle glaucoma: a meta-analysis. Diabet Med. 2004;21(6):609-14.

Endereço para Correspondência: Jacqueline Coblentz

Rua Visconde de Silva, $n^{\circ}$ 52/703

CEP 22271-090 - Rio de Janeiro (RJ), Brasil

e-mail: j_coblentz@yahoo.com 